Nonlinear Processes in Geophysics (2002) 9: 25-29

Nonlinear Processes

in Geophysics

(C)European Geophysical Society 2002

\title{
Spiky parallel electrostatic ion cyclotron and ion acoustic waves
}

\author{
R. V. Reddy ${ }^{1}$, G. S. Lakhina ${ }^{1}$, N. Singh ${ }^{2}$, and R. Bharuthram ${ }^{3}$ \\ ${ }^{1}$ Indian Institute of Geomagnetism, Colaba, Mumbai - 400005, India \\ ${ }^{2}$ Department of Electrical and Computer Engineering, University of Alabama, Huntsville, AL 35899, USA \\ ${ }^{3}$ M. L. Sultan Technikon, P.O. Box 1334, Durban 4000, South Africa
}

Received: 22 February 2001 - Revised: 14 May 2001 - Accepted: 18 May 2001

\begin{abstract}
One of the interesting observations from the FAST satellite is the detection of strong spiky waveforms in the parallel electric field in association with ion cyclotron oscillations in the perpendicular electric fields. We report here an analytical model of the coupled nonlinear ion cyclotron and ion-acoustic waves, which could explain the observations. Using the fluid equations for the plasma consisting of warm electrons and cold ions, a nonlinear wave equation is derived in the rest frame of the propagating wave for any direction of propagation oblique to the ambient magnetic field. The equilibrium bulk flow of ions is also included in the model to mimic the field-aligned current. Depending on the wave Mach number $M$ defined by $M=V / C_{s}$ with $V$ and $C_{s}$ being the wave phase velocity and ion-acoustic speed, respectively, we find a range of solutions varying from a sinusoidal wave form for small amplitudes and low $M$ to sawtooth and highly spiky waveforms for nearly parallel propagation. The results from the model are compared with the satellite observations.
\end{abstract}

\section{Introduction}

The electrostatic ion cyclotron (EIC) mode is one of the low frequency eigen modes of a magnetized plasma (D'Angelo and Motley, 1962). These waves are studied due to their importance in the heating of plasmas (Kelley et al., 1975). The EIC waves are found to be unstable to current-driven instabilities in the auroral region (Kindel and Kennel, 1971). The measurements performed by the S3-3 (Mozer et al., 1977), ISEE-1 (Cattell et al., 1991), Viking (Andre et al., 1987), Polar (Mozer et al., 1997) and FAST (Ergun et al., 1998) satellites have shown that EIC waves are frequently observed in the auroral magnetosphere at altitudes between $3000 \mathrm{~km}$ to $8000 \mathrm{~km}$ and beyond.

The nonlinear steepening of the time domain perpendicular electric field structures in an EIC wave has been stud-

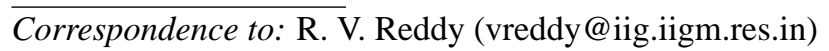

ied by Temerin et al. (1979). In their model, they observed spiky structures in the perpendicular electric field for large Mach numbers. The nonlinear EIC and ion acoustic waves in a low $\beta$ magnetized plasma were studied by Lee and Kan (1981). They have presented a unified formulation for the nonlinear electrostatic ion cyclotron and ion acoustic waves. In an earlier study, Shukla and Yu (1978) have investigated the oblique propagation of finite amplitude ion acoustic solitons in a magnetized plasma. Their model was extended to include the effects of the nonlinear ion polarization drift by Yu et al. (1980). Recently, Jovanovic and Shukla (2000) have presented a nonlinear model for coherent electric field structures in the magnetosphere by taking into account the low frequency ion dynamics.

From the Polar data, Mozer et al. (1997) have observed that the perpendicular electric field exhibits spiky structures with amplitudes about $400 \mathrm{mV} / \mathrm{m}$ and repetition rates in the range of the local ion cyclotron frequency. In addition, bipolar structures and wave packets were observed in the parallel electric field. Recently, wave observations by the FAST satellite (Ergun et al., 1998) also revealed the existence of nonlinear, time-domain structures associated with parallel electric fields. These parallel electric field structures carry large potentials and are associated with ion cyclotron waves. In Fig. 1, we show an event from the FAST satellite taken from Ergun et al. (1998), which exhibits the time development of nonlinear ion cyclotron wave. The upper curve in Fig. 1 shows bipolar electric field structures along the ambient magnetic field direction with amplitude $\sim 700 \mathrm{mV} / \mathrm{m}$. The lower part of Fig. 1 displays the perpendicular electric field structures of the ion cyclotron $(f \sim 200 \mathrm{~Hz})$ wave, which do not contain the bipolar structures. From Fig. 1, we infer that the frequency of the parallel propagating electric field structure is $\sim 50 \mathrm{~Hz}$ and is four times less than the frequency of electric field structure of the perpendicularly propagating ion cyclotron wave.

The existence of spiky parallel electric field oscillations at the ion cyclotron frequency have also been reported from numerical simulations in which diverging and converging 


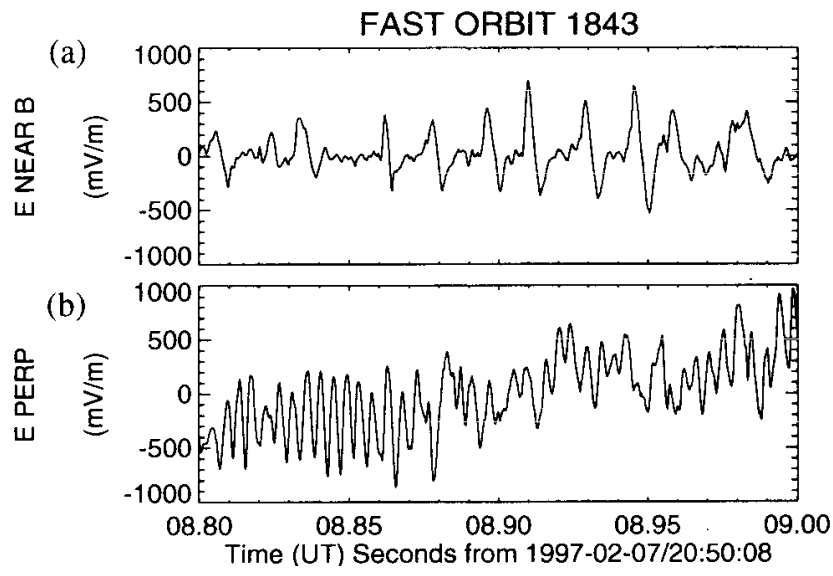

Fig. 1. Parallel and perpendicular electric field structures observed by FAST satellite (taken from Ergun et al., 1998).

electrostatic shocks were generated by field-aligned current sheets (Singh et al., 1987). In the simulation $E_{\|}$was spiky as in Fig. 1, without any such spiky structure in the perpendicular component of the field. Despite the observations from FAST (Ergun et al., 1998) and simulation results (Singh et al., 1987) on the spiky parallel electric fields without spikes in $E_{\perp}$, no satisfactory attempt has been made to explain such structures. The theoretical work of Temerin et al. (1979) is limited to explaining the spiky nature in $E_{\perp}$ as observed from S3-3 (Mozer et al., 1977). However, the observation of parallel oscillations at the ion acoustic frequency (or less than ion cyclotron frequency) with a signature of ion cyclotron oscillations in the perpendicular field (see Fig. 1) has remained unexplained. The linear analysis shows that for parallel propagation, electrostatic ion wave dispersion relation can support both ion acoustic and ion cyclotron waves (Nicholson, 1983). We show here that the nonlinear coupling between the ion acoustic mode and ion cyclotron oscillations lead to the generation of parallel electric fields which can be highly spiky and these spikes have various periods ranging from ion cyclotron to ion acoustic. The equilibrium flow of ions is included in the model to account for the field-aligned current.

\section{Model}

For our model, we consider a homogeneous magnetized plasma system consisting of cold ions and electrons. The finite amplitude ion cyclotron/ion acoustic waves are propagating in the $x$ direction at an angle $\theta$ to the magnetic field $\boldsymbol{B}_{\mathbf{0}}$, which is assumed to be in the $(x, z)$-plane. The basic set of fluid equations for this system is given by

$$
\begin{aligned}
& \frac{\partial n_{i}}{\partial t}+\frac{\partial\left(n_{i} v_{i x}\right)}{\partial x}=0 \\
& \frac{\partial v_{i x}}{\partial t}+v_{i x} \frac{\partial v_{i x}}{\partial x}=-\frac{e}{m_{i}} \frac{\partial \phi}{\partial x}+\Omega_{i} v_{i y} \sin \theta
\end{aligned}
$$

$$
\begin{aligned}
& \frac{\partial v_{i y}}{\partial t}+v_{i x} \frac{\partial v_{i y}}{\partial x}=\Omega_{i} v_{i z} \cos \theta-\Omega_{i} v_{i x} \sin \theta \\
& \frac{\partial v_{i z}}{\partial t}+v_{i x} \frac{\partial v_{i z}}{\partial x}=-\Omega_{i} v_{i y} \cos \theta,
\end{aligned}
$$

where $n_{i}$ is the ion density, $v_{i x}, v_{i y}$ and $v_{i z}$ are the components of the ion velocity along the $x, y$, and $z$ directions, respectively, $\Omega_{i}$ is the ion cyclotron frequency and $\phi$ is the electrostatic potential of the waves. Ion thermal pressure is neglected $\left(T_{e} \gg T_{i}\right)$. Since we are studying low frequency phenomena $\left(\omega \ll \Omega_{e}\right)$, the electrons are assumed to be in electrostatic equilibrium. Their density is then given by the Boltzmann distribution, $n_{e}=n_{0} \exp \left(e \phi / T_{e}\right)$, where $n_{0}$ is the ambient density. Our system of equations is closed with the quasi-neutrality condition $n_{i}=n_{e}$, which is valid for low frequency studies.

We look for solutions of Eqs. (1)-(4) that depend on $x$ and $t$ through a variable $\eta=(x-V t)\left(\Omega_{i} / V\right)$, where $V$ is the phase velocity of the wave. Integrating Eq. (1) and using the conditions $n_{i}=n_{0}$ and $v_{x}=v_{0}$ at $\eta=0$ yield

$n_{i} v_{x}^{\prime}=-n_{0}\left(V-v_{0}\right)$,

where $v_{x}^{\prime}=v_{x}-V$.

Combining Eqs. (2)-(3) gives

$$
\begin{aligned}
v_{x}^{\prime} & \frac{\partial}{\partial \eta}\left(\frac{1}{2} \frac{\partial v_{x}^{\prime 2}}{\partial \eta}+\frac{e}{m_{i}} \frac{\partial \phi}{\partial \eta}\right)+v_{x} V^{2} \sin ^{2} \theta \\
& =v_{z} V^{2} \cos \theta \sin \theta .
\end{aligned}
$$

Combining Eqs. (2)-(4) yields

$$
v_{x}^{\prime} \sin \theta \frac{\partial v_{z}}{\partial \eta}+v_{x}^{\prime} \cos \theta \frac{\partial v_{x}}{\partial \eta}+\cos \theta\left(\frac{e}{m_{i}}\right) \frac{\partial \phi}{\partial \eta}=0
$$

The quasi-neutrality condition gives

$v_{x}^{\prime}=-\left(V-v_{0}\right) e^{\left(-e \phi / T_{e}\right)}$.

By eliminating $v_{x}$ and $v_{z}$ from Eqs. (6)-(8) and introducing the dimensionless quantities $\psi=e \phi / T_{e}, M=V / C_{s}$ and $\delta=v_{0} / C_{s}$, where $C_{s}=\left(T_{e} / m_{i}\right)^{1 / 2}$ is the ion acoustic speed, we obtain

$$
\begin{array}{r}
\frac{d}{d \eta}\left(e^{-\psi} \frac{d^{2} \psi}{d \eta^{2}}+\frac{M_{A}^{2} e^{-\psi}}{2} \frac{d^{2} e^{-2 \psi}}{d \eta^{2}}\right) \\
=-\frac{M^{2} \cos ^{2} \theta e^{\psi}}{M_{A}^{2}} \frac{d \psi}{d \eta}-M^{2} \frac{d e^{-\psi}}{d \eta},
\end{array}
$$

where $M_{A}=M-\delta$.

Integrating Eq. (9) and using the conditions $\psi=0$, $d \psi / d \eta=E_{0}$ and $d^{2} \psi / d \eta^{2}=0$ at $\eta=0$, we obtain

$$
\chi_{1}(\psi) \frac{d^{2} \psi}{d \eta^{2}}-\chi_{2}(\psi)\left(\frac{d \psi}{d \eta}\right)^{2}+\chi_{3}(\psi)=0,
$$

where

$\chi_{1}(\psi)=e^{-2 \psi}-\frac{1}{M_{A}^{2}}$ 


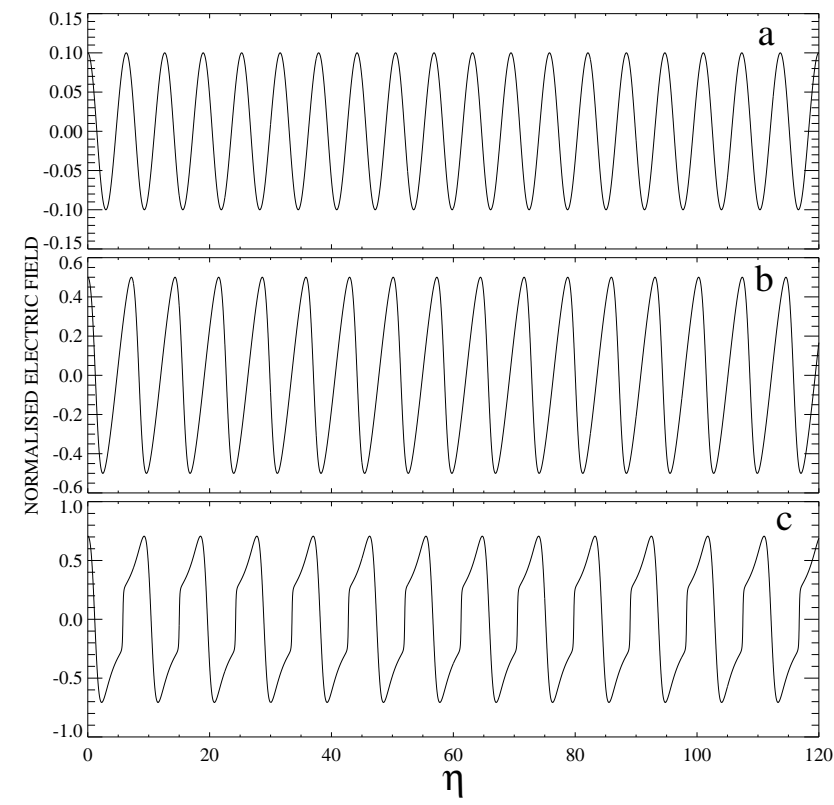

Fig. 2. Numerical solution of the normalized parallel electric field $(=\partial \psi / \partial \eta)$ of nonlinear electrostatic ion cyclotron and ion acoustic waves for the parameters $\theta=2^{\circ}, M=0.2, \delta=0$ and $E_{0}=0.1$ (a), 0.5 (b), and 0.7065 (c)

$\chi_{2}(\psi)=2 e^{-2 \psi}$

$$
\begin{aligned}
& \chi_{3}(\psi)=e^{\psi}\left(\frac{M^{2}}{M_{A}^{2}}+2 E_{0}^{2}\right) \\
& \frac{M^{2}}{M_{A}^{2}}-\frac{M^{2} \cos ^{2} \theta e^{\psi}}{M_{A}^{4}}\left(e^{\psi}-1\right) .
\end{aligned}
$$

In Eq. (10), $d \psi / d \eta$ is the normalized electric field.

Equation (10) describes the nonlinear evolution of both ion cyclotron and ion acoustic waves. For $\theta=90^{\circ}, v_{0}=0$ and $E_{0}=0$, we recover from Eq. (10) the perpendicularly propagating EIC wave (Temerin et al., 1979), as a special case. When the bulk ion flow $v_{0}=0$ and $E_{0}=0$, Eq. (10) reduces to Eq. (10) of Lee and Kan (1981), which corresponds to the oblique propagation of ion cyclotron and ion aoustic modes.

In the linear regime, we find that Eq. (2) decouples from Eqs. (3) and (4), giving the ion acoustic wave and local (nonpropagating) ion cyclotron oscillations when $\theta=0$. But when the nonlinear effects are significant, then Eqs. (2),(3) and (4) are no longer decoupled and the ion acoustic and ion cyclotron modes are coupled through the convective terms $v_{i x}\left(\partial v_{i y} / \partial x\right)$ and $v_{i x}\left(\partial v_{i z} / \partial x\right)$.

\section{Numerical results}

We have numerically solved the nonlinear evolution Eq. (10). In view of the observations of spiky parallel electric fields shown in Fig. 1, here we focus the analysis on nearly parallel propagating nonlinear ion acoustic and ion cyclotron waves. However, we point out that our model can also be used to

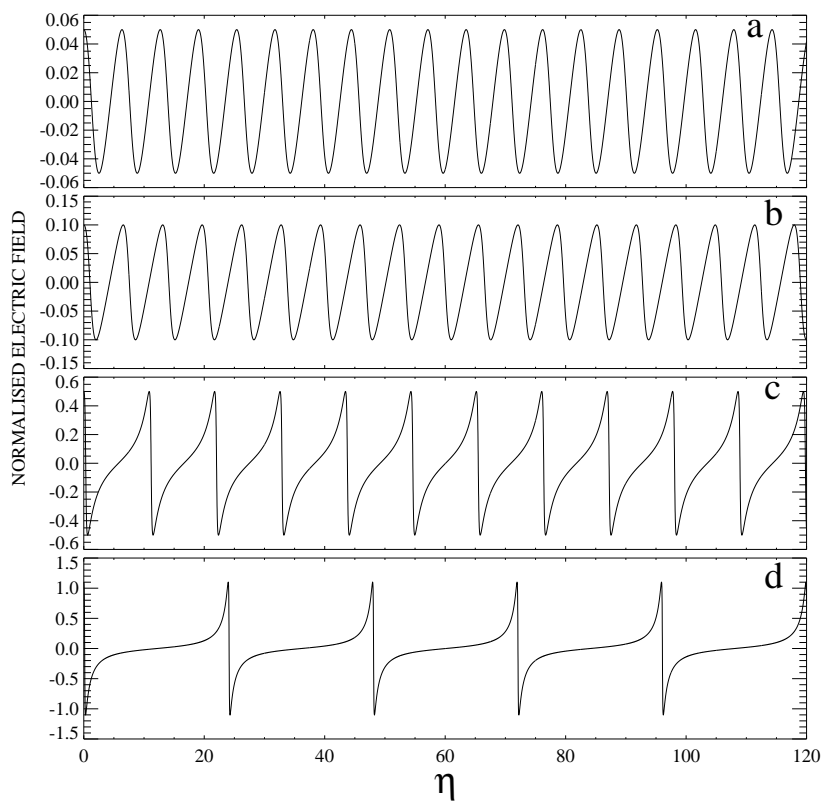

Fig. 3. Here $M=1.25$ and $E_{0}=0.05$ (a), 0.10 (b), 0.5 (c), and 1.1 (d). All other fixed parameters are as in Fig. 2.

deduce the propagating modes for any arbitrary direction of propagation including the perpendicular spiky electric field structures of the nonlinear ion cyclotron waves reported by Temerin et al. (1979). In Fig. 1, the period of oscillation of the wave propagating nearly parallel to $\boldsymbol{B}_{\mathbf{0}}$ is found to be about four times the period of the perpendicularly propagating mode. The frequency of the parallel spikes in Fig. 1 is found to be $\sim 50 \mathrm{~Hz}$, while the ion cyclotron frequency is about $200 \mathrm{~Hz}$.

Our numerical studies show that the nonlinear wave Eq. (10) has a strong dependence on the driving amplitude, $E_{0}$, Mach number, $M$, and the bulk flow velocity, $v_{0}$. In our analysis, we perform a parametric study by varying the Mach number, the driving amplitude and the bulk flow velocity on a nearly parallel propagating wave $\left(\theta=2^{\circ}\right)$. In Fig. 2, we keep $v_{0}=0$ and consider a small Mach number, that is $M=0.2$ and varied the driver, $E_{0}$. The figure displays the normalized electric field $E$, given by $E=\partial \psi / \partial \eta$. Since $\theta=2^{o}$, this field is essentially the parallel electric field.

The period of oscillations is given by $\Delta \eta=\left(\Omega_{i} / V\right) \Delta x-$ $\Omega_{i} \Delta t$, typically for $\Delta x=0, \Delta \eta=\left|\Omega_{i} \Delta t\right|$. For the relatively small driving amplitude of 0.1 (Fig. 2a), we observe that the model exhibits ion cyclotron oscillations; these oscillations have a period of $\Delta \eta \cong 6.3$ or $\Delta t \cong 6.3 \Omega_{i}^{-1}=1 \tau_{c i}$, where $\tau_{c i}=2 \pi / \Omega_{i}$ is the ion cyclotron period. As the driving amplitude increases, we finally obtain a sawtooth waveform with a periodicity of $\Delta \eta \cong 10$ when $E_{0} \cong 0.71$, which yields a period of about one and half times the ion cyclotron period (Fig. 2c). Since the period of these spiky structures is larger than the ion cyclotron period, we interpret this waveform to be the driven ion acoustic mode.

Figure 3 shows the variation of the parallel electric field for Mach number, $M=1.25, v_{0}=0$ and for different driver 


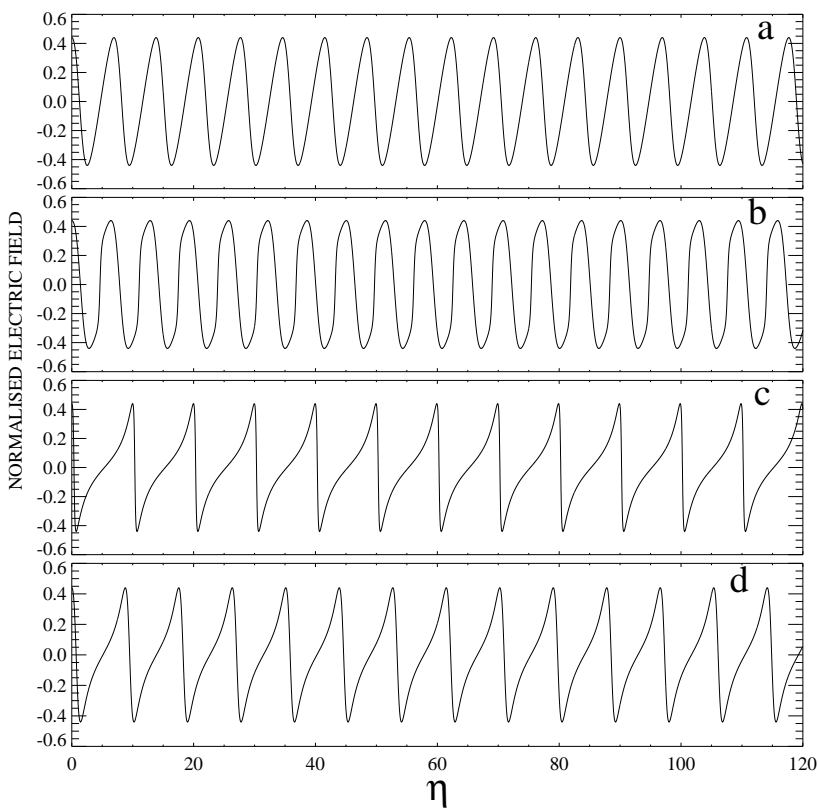

Fig. 4. Variation of the normalized electric field for $E_{0}=0.44$ and $M=0.2$ (a), 0.5 (b), 1.25 (c) and 4.5 (d). Other fixed parameters are as in Fig. 2.

strengths. Here we observe that the $E_{\|}$shows ion cyclotron oscillations for small $E_{0}$ with a period $\Delta \eta \cong 6.3$ or $\Delta t \cong \tau_{c i}$ (Fig. 3a) and with increasing driver strength the cyclotron oscillations tend to steepen (Fig. 3b). As the driving amplitude increases, we obtain a driven ion acoustic mode which exhibits waveforms somewhat similar to a sawtooth type with a period, $\Delta \eta \cong 12$ (Fig. 3c). Further increase in driver strength yields a driven ion acoustic oscillation with highly spiky bipolar structure in the waveform with a periodicity, $\Delta \eta \cong 24$, which yields a period of about four times the ion cyclotron period (Fig. 3d) and has a great deal of similarity with the observed structure shown in Fig. 1a.

In Fig. 4, we have varied the Mach number, $M$ by keeping $v_{0}=0$ and the driver strength constant. When $M$ is small, $E_{\|}$exhibits the oscillations in the ion cyclotron period range and the waveform of these oscillations tends to be of the sawtooth type. With an increase in Mach number, the $E_{\|}$ shows a sawtooth structure with a period of approximately $\Delta t \cong \Delta \eta \Omega_{i}^{-1} \simeq 10 \Omega_{i}^{-1} \approx 1.7 \tau_{c i}$. Figure 4 shows that as $M$ increases further, i.e. when $M>1$, the normalized period $(\Delta \eta)$ of the spiky fields decreases (Figs. $4 \mathrm{c}$ and $4 \mathrm{~d}$ ).

Figure 5 shows the variation of the parallel electric field for $M=1.25, E_{0}=1.1$ and for different $\delta$. Here we notice that the period of the spiky structures decreases as $\delta$ varies from -0.2 to 0.2 . Therefore, the ion flow parallel (antiparallel) to $\boldsymbol{B}_{\mathbf{0}}$ would decrease (increase) the period of the driven spiky structure.

We infer from our calculations that to obtain highly spiky ion acoustic parallel electric field structures, the system must be driven quite strongly. In the above discussion we have mainly shown that the fluid equations do permit highly spiky solutions even for nearly parallel propagation. We compare

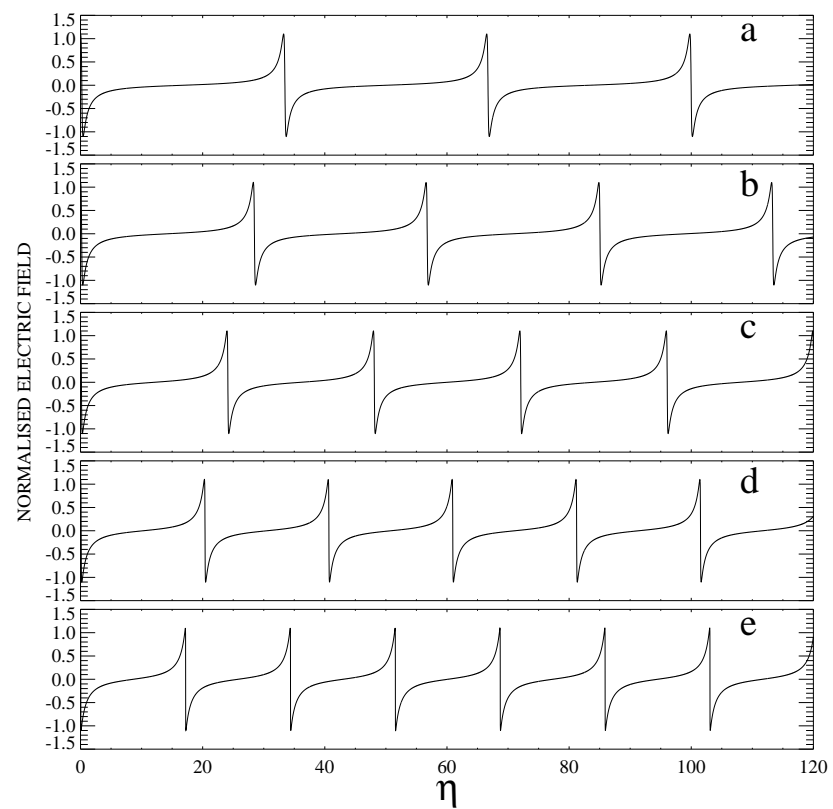

Fig. 5. The normalized electric field for $E_{0}=1.1, M=1.25$ and $\delta=-0.2$ (a), -0.1 (b), 0.0 (c), 0.1 (d), 0.2 (e). All other fixed parameters are as in Fig. 2.

now the strength of the spiky fields obtained from our calculations and that measured by satellites, as shown, for example, in Fig. 1a. The unnormalized electric field can be written as

$$
\begin{aligned}
E= & -\frac{\partial \phi}{\partial x}=-\left(\Omega_{i} / C_{s}\right)\left(T_{e} / e\right)\left(\frac{1}{M} \partial \psi / \partial \eta\right) \\
& =\left(T_{e} / e \rho_{i s}\right)\left(\frac{1}{M} \partial \psi / \partial \eta\right),
\end{aligned}
$$

where $\rho_{i s}=C_{s} / \Omega_{i}$ is the ion Larmor radius at the ionacoustic speed $C_{s}$, and $T_{e}$ is in energy units. The energy of electrons in the upward current region just before the event of Fig. 1 can be roughly estimated as $T_{e}=10-100 \mathrm{eV}$ (Ergun et al., 1998). This would give an ion acoustic speed, $C_{s} \cong 50-150 \mathrm{~km} / \mathrm{s}$, and the wave phase velocity $V=$ $M C_{S}=10-600 \mathrm{~km} / \mathrm{s}$ for the range of $M$ considered in this paper. Also the value of the ambient magnetic field $B_{0} \approx 13000 \mathrm{nT}$, gives $\Omega_{i}=200 \times 2 \pi \mathrm{rad} / \mathrm{s}$. The scale size of the spiky structures from our model are estimated as $\sim(1.5-15) \rho_{i s} \cong 75-1800 \mathrm{~m}$. Our model predicts that the repetition frequency of the spiky parallel electric fields is such that generally $\omega_{r}<\Omega_{i}$, and it is possible to have $\omega_{r} \sim \Omega_{i} / 4$ in accordance with the FAST observations. Furthermore, for the above parameters, we find that $E_{\|}$as calculated from Eq. (14) can range from $\sim 50 \mathrm{mV} / \mathrm{m}$ to a few $\mathrm{V} / \mathrm{m}$. In Fig. 1 the measured value is about $700 \mathrm{mV} / \mathrm{m}$, which is within the predicted range of values.

\section{Conclusions}

FAST and Polar observations in the auroral magnetosphere have shown that the nonlinear, time domain structures as- 
sociated with the parallel electric field exhibit spiky wave forms. These quasi-static, parallel electric field structures are thought to be responsible for particle acceleration in the auroral acceleration region. The model presented in this study could explain the generation mechanism of these parallel propagation structures which show sawtooth or spiky wave forms. Observations by FAST (as shown in Fig. 1) can be explained naturally by assuming a mixture of near parallel and near perpendicular propagating wave modes. The free energy source for these waves could be either electron/ion beams or the field-aligned currents. The present model can generate various wave-forms ranging from ion cyclotron to ion acoustic modes, depending on the initial driver and the Mach number. We have not considered growth or damping of these waves. It would be interesting to study the stability of these structures. We have neglected the ion temperature effects and treated ions as cold fluid. The inclusion of finite ion temperature will give rise to a dispersive effect, which may tend to broaden the structures. Therefore, the present model can be applied where $T_{e} \gg T_{i}$, such as the auroral acceleration region. From our model the estimated length scales of the nonlinear structures are of the order of $1.5-15 \rho_{i s}$. This implies that the length scales are much larger than the ion gyro radius and hence the finite Larmor radius effects are not expected to be important, and the use of uniform magnetized plasma fluid model is justified. However, the finite Larmor radius effect of ions could introduce dispersion and a new nonlinear term which may lead to cylindrically symmetric electron hole as discussed by Jovanovic and Shukla (2000). We have presented a simple model for the auroral plasma which takes care of the field-aligned current. The model can be generalized to include a background plasma and multi-beams to make it even closer to the observations.

Acknowledgements. N. Singh acknowledges support from NSF grant ATM-9814571 and R. Bharuthram from the NRF, South Africa. They both thank the entire staff of the Indian Institute of Geomagnetism for their warm hospitality during their visits.

\section{References}

Andre, M., Koskinen, H., Gustafsson, G., and Lundin, R.: Ion waves and upgoing ion beams observerd by the Viking satellite, Geophys. Res. Lett., 14, 463-466, 1987.
Cattell, C. A., Mozer, F. S., Roth, I., Anderson, R. R, Elphic, R. C., Lennartsson, W., and Ungstrup, E.: ISEE 1 Observations of EIC waves in Association with Ion Beams on Auroral field Lines From $\sim 2.5$ to $4.5 R_{E}$, J. Geophys. Res., 96, 11421-11439, 1991.

D'Angelo, N. and Motley, R. W.: Electrostatic Oscillations near the Ion Cyclotron Frequency, Phys. Fluids, 5, 633-634, 1962.

Ergun, R. E., Carlson, C. W., McFadden, J. P., Mozer, F. S., Delory, G. T., Peria, W., Chaston, C. C., Temerin, M., Elphic, R., Strangeway, R., Pfaff, R., Cattell C. A., Klumpar D., Shelley, E., Peterson, W., Moebius, E., and Kistler, L.: FAST satellite observations of electric field structures in the auroral zone, Geophys. Res. Lett., 25, 2025-2028, 1998.

Jovanovic, D. and Shukla, P. K.: Nonlinear Model for Coherent Electric Field Structures in the Magnetosphere, Phys. Rev. Lett., 84, 4373-4376, 2000.

Kelley, M. C., Bering, E. A., and Mozer, F. S.: Evidence that the electrostatic ion cyclotron instabilty is saturated by ion heating, Phys. Fluids, 18, 1590-1591, 1975.

Kindel, J. M. and Kennel, C. F.: Topside current instabilities, J. Geophys. Res., 76, 3055-3078, 1971.

Lee, L. C. and Kan, J. R.: Nonlinear ion-acoustic waves and solitons in a magnetized plasma, Phys. Fluids, 24, 430-433, 1981.

Mozer, F. S., Carlson, C. W., Hudson, M. K., Torbert, R. B., Parady, B., Yatteau, J., and Kelley, M. C.: Observations of paired electrostatic shocks in the polar magnetosphere, Phys. Rev. Lett.,38, 292-295, 1977.

Mozer, F. S., Ergun, R., Temerin, M., Cattell, C., Dombeck, J., and Wygant, J.: New Features of Time Domain Electric-Field Structures in the Auroral Acceleration Region, Phys. Rev. Lett., 79, 1281-1284, 1997.

Nicholson, D. R.: Introduction to Plasma Theory, John Wiley, New York, 1983.

Singh, N., Thiemann, H., and Schunk, R. W.: Simulation of Auroral Plasma Processes: Electric Fields, Waves and Particles, Planet. Space Sci., 35, 353-395, 1987.

Shukla, P. K. and Yu, M. Y.: Exact solitary ion acoustic waves in a magnetoplasma, J. Mathematical Phys., 19, 2506-2508, 1978.

Temerin, M., Woldorff, M., and Mozer, F. S.: Nonlinear Steepening of the Electrostatic Ion Cyclotron Wave, Phys. Rev. Lett., 43, 1941-1943, 1979.

Yu, M. Y., Shukla, P. K., and Bujarbarua, S.: Fully nonlinear ionacoustic solitary waves in a magnetized plasma, Phys. Fluids, 23, 2146-2147, 1980. 